\title{
Prevalence and distribution pattern of nodal metastases in advanced ovarian cancer
}

\author{
CORNELIA BACHMANN ${ }^{1}$, ROBERT BACHMANN ${ }^{2}$, BERNHARD KRAEMER ${ }^{1}$, SARA YVONNE BRUCKER ${ }^{1}$, \\ ANETTE STAEBLER $^{3}$, FALKO FEND $^{3}$, RALF ROTHMUND $^{1}$ and DIETHELM WALLWIENER ${ }^{1}$ \\ ${ }^{1}$ Department of Gynecology, University of Tübingen, D-72070 Tübingen; ${ }^{2}$ Department of General, Visceral and Transplant \\ Surgery, University Hospital of Tübingen; ${ }^{3}$ Department of Pathology, University of Tübingen, D-72076 Tübingen, Germany
}

Received April 18, 2016; Accepted June 22, 2016

DOI: $10.3892 / \mathrm{mco} .2016 .982$

\begin{abstract}
The objective of this study was to examine the relevance of pelvic and para-aortic lymph node involvement and the tumour characteristics affecting nodal metastases and survival in primary advanced ovarian cancer. A total of 130 consecutive patients were retrospectively investigated. All the patients received stage-related surgery with pelvic and para-aortic lymphadenectomy. The median follow-up was 53.5 months. The clinicopathological parameters and distribution pattern of nodal metastases were evaluated. Lymph node metastases were detectable in $74.62 \%$ of the cases. Overall, both pelvic and para-aortic nodes were affected in $35.9 \%$ of the patients, whereas $13.3 \%$ had metastases only in the pelvic and $13.3 \%$ only in the para-aortic lymph nodes. Histological grade $1 / 2$ and 3 , serous and endometrioid histology were independent predictors of nodal metastasis. Serous and endometrioid cancers have shown a predilection for metastasis to the pelvic lymph nodes alone, both to the pelvic and the para-aortic nodes, or the para-aortic nodes alone. Overall survival was significantly positively affected by serous histology with positive nodes $(\mathrm{P}=0.043)$. It is crucial to investigate the risk factors and metastatic patterns of such patients in a multicenter analysis to evaluate individual subgroups. Prospective studies are required to investigate the prognostic effect of lymphadenectomy in advanced ovarian cancer and its association with histology and distribution pattern of nodal metastasis.
\end{abstract}

\section{Introduction}

The role of systematic lymphadenectomy in primary advanced ovarian cancer and its prognostic effect remain controversial and there are currently no available results from randomised

Correspondence to: Dr Cornelia Bachmann, Department of Gynecology, University of Tübingen, 7 Calwer Street, D-72070 Tübingen, Germany

E-mail: cornelia.bachmann@med.uni-tuebingen.de

Key words: nodal involvement, advanced ovarian cancer, lymphadenectomy, histology, tumour characteristics controlled studies. There is an increased tendency for nodal invasion in advanced ovarian cancer $(1,2)$. Panici et al described a positive prognostic effect of systematic lymphadenectomy on progression-free survival (PFS) compared with resection of bulky nodes, but not on overall survival (OS) (3). By contrast, other studies reported a positive prognostic effect on OS after systematic lymphadenectomy in this subgroup (4). Therefore, it remains unclear whether complete staging with systematic lymphadenectomy and its associated morbidity is beneficial in this subgroup. Little is known on the extent of lymph node metastases, or which lymph node region is affected depending on histological subtype. The objective of this study was to delineate the prevalence and distribution pattern of pelvic and para-aortic lymph node metastases and the tumour characteristics, such as histological subtype, affecting survival in primary advanced ovarian cancer.

\section{Patients and methods}

Patients. A total of 130 consecutive patients with primary International Federation of Gynecology and Obstetrics (FIGO) stage IIIC/IV advanced epithelial ovarian cancer who were treated at the Department of Gynecology, University of Tübingen (Tübingen, Germany), were retrospectively analysed. Each patient underwent surgical staging following hysterectomy, bilateral adnexectomy, supracolic omentectomy and cytoreduction, as indicated. Pelvic and para-aortic lymphadenectomy was performed up to the level of the renal vessels in all patients with optimally cytoreduced ovarian cancer. All the patients were treated with an adjuvant standard platinum-based chemotherapy, according to the AGO guidelines (http://www.ago-online.de/de/infothek-fuer-aerzte/leitlinienempfehlungen/ovar/). A total of 130 patients met the inclusion criteria and were enrolled. The affected region of nodal metastasis (pelvic or para-aortic) and the clinicopathological factors that were associated with nodal involvement were identified (Table I). All the patients provided informed consent for data acquisition prior to their inclusion in the evaluation. For evaluation of the affected lymph node region depending on histology, data on 2 patients were missing and were not further evaluated in this subgroup.

The present study was conducted in compliance with the current national laws and the principles laid down in 
the Declaration of Helsinki and was approved by the Ethics Committee of the University of Tübingen (no. 244/2015R).

Histology. All surgical tissues were examined by a pathologist and the final pathology reports were obtained. All the surgical pathology samples were examined by a gynaecological pathologist and each diagnosis was reviewed and classified as benign or malignant. The histological diagnosis was classified according to the FIGO staging guidelines (5).

Follow-up. Follow-up data were collected at presentation in our outpatient department. The mean follow-up time was 53.5 months.

Statistical analysis. Statistical analyses (multivariate) were performed using PASW Statistics, version 22 (PASW Inc., Chicago, IL, USA). The Student's $t$-test was used for testing significant differences between the examined groups. The significance level was set at $\mathrm{P}<0.05$.

\section{Results}

Patient characteristics. Of the 130 enrolled patients, the majority $(n=108)$ were classified as FIGO stage IIIC and the remaining $(n=22)$ as FIGO stage IV. The patient characteristics are summarised in Table I. Overall, the most common histological grade was $3(54.62 \%)$ and the most common histological type was serous $(85.38 \%)$, followed by the undifferentiated $(4.62 \%)$ and endometrioid (6.2\%) subtypes (Table I); the mucinous and clear cell subtypes were rarely detected (Table I). Lymph node metastases were detected in $74.62 \%$ of the cases (Table I).

Considering the affected lymph node regions, the following were observed: The most frequent pattern was invasion of both the pelvic and para-aortic nodes (35.9\% of the patients); isolated involvement of the pelvic or para-aortic lymph nodes was detected in $13.3 \%$ of the patients, whereas 13 patients had positive pelvic nodes with unknown status of the para-aortic nodes and 2 patients had positive para-aortic nodes with unknown status of the pelvic nodes (Table I).

On multivariate analysis, histological grade $1 / 2$ and 3 , and serous and endometrioid histology were found to be independent predictors of nodal metastasis (Table II). Serous histology is most commonly associated with nodal metastasis; however, patients with advanced ovarian cancer with mucinous, clear cell and endometrioid histology also had node metastases (Table II). Considering the limited number of cases, mucinous cancers rarely led to nodal metastasis, whereas metastasis was detected in $84.2 \%(n=16)$ of non-serous cancers (Table II).

Serous cancers were associated with metastasis to the pelvic lymph nodes alone, the para-aortic nodes alone, and both the pelvic and para-aortic nodes; a combined involvement of the pelvic and para-aortic nodes was the most frequent pattern in this subgroup (Table III). Of the patients with non-serous cancers, $27.77 \%(n=5)$ exhibited involvement of the pelvic and para-aortic nodes (Table III). Patients with endometrioid histology had exhibited metastases to the pelvic nodes alone, or to both the pelvic and para-aortic nodes; isolated involvement of the para-aortic lymph nodes was the most frequent pattern detected in this subgroup (Table III). Among patients with
Table I. Clinicopathological characteristics of patients with FIGO stage IIIC/IV primary ovarian cancer.

\begin{tabular}{|c|c|}
\hline Characteristics & $\mathrm{n}(\%)$ \\
\hline \multicolumn{2}{|l|}{ Histologic grade } \\
\hline $1 / 2$ & $59(45.38)$ \\
\hline 3 & $71(54.62)$ \\
\hline \multicolumn{2}{|l|}{ Histology } \\
\hline Serous & $111(85.38)$ \\
\hline Mucinous & $3(2.3)$ \\
\hline Clear cell & $2(1.5)$ \\
\hline Endometrioid & $8(6.2)$ \\
\hline Undifferentiated & $6(4.62)$ \\
\hline \multicolumn{2}{|l|}{ Node involvement } \\
\hline No & $33(25.38)$ \\
\hline $\mathrm{N}+$ & $97(74.62)$ \\
\hline \multicolumn{2}{|l|}{ R-status } \\
\hline $\mathrm{R} 0$ (0 mm) & $39(30.0)$ \\
\hline $\mathrm{R}>0-<10 \mathrm{~mm}$ & $69(53.07)$ \\
\hline $\mathrm{R} \geq 10 \mathrm{~mm}$ & $22(16.93)$ \\
\hline \multicolumn{2}{|c|}{ Region with node involvement $^{\mathrm{a}}(\mathrm{n}=128)$} \\
\hline Pelvic -/paraaortic - & $33(25.78)$ \\
\hline Pelvic + /paraaortic - & $17(13.3)$ \\
\hline Pelvic +/paraaortic + & $46(35.9)$ \\
\hline Pelvic -/paraaortic + & $17(13.3)$ \\
\hline Pelvic + /paraaortic $\mathrm{x}$ & $13(10.16)$ \\
\hline Pelvic x/paraaortic + & $2(1.56)$ \\
\hline
\end{tabular}

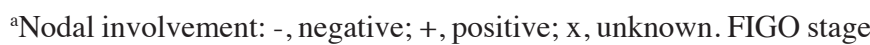
IIIC, n=108; FIGO stage IV, n=22. FIGO, international federation of gynecology and obstetrics.

Table II. Evaluation of lymph node metastases in correlation with clinicopathological parameters, such as histology (serous vs. non-serous) and histological grade in 130 FIGO stage IIIC/IV ovarian cancer patients.

\begin{tabular}{lcc}
\hline Parameters & $\mathrm{N} 0, \mathrm{n}(\%)$ & $\mathrm{N}+, \mathrm{n}(\%)$ \\
\hline $\begin{array}{l}\text { Histologic grade } \\
1 / 2\end{array}$ & $21(16.2)$ & $38(29.2)$ \\
3 & $12(9.2)$ & $59(45.4)$ \\
Histology & & \\
Serous & $30(23.1)$ & $81(62.3)$ \\
Mucinous & $2(1.5)$ & $1(0.8)$ \\
Clear cell & $0(0)$ & $2(1.5)$ \\
Endometrioid & $0(0)$ & $8(6.2)$ \\
Undifferentiated & $0(0.8)$ & $5(3.8)$ \\
\hline
\end{tabular}

Nodal involvement: -, negative; +, positive; x, unknown. FIGO, international federation of gynecology and obstetrics.

mucinous cancers $(n=3), 1$ had metastatic pelvic nodes and 2 had negative nodes (Table III). Of the 2 patients with the clear 
Table III. Description of lymph node metastases in correlation with histological subtype (serous vs. non-serous) and the affected region (pelvic/para-aortic) in 128 patients with FIGO stage IIIC/IV primary ovarian cancer.

\begin{tabular}{|c|c|c|c|c|c|c|}
\hline Histology & $\begin{array}{l}\text { Pelvic-/para- } \\
\text { aortic - (n) }\end{array}$ & $\begin{array}{l}\text { Pelvic +/para- } \\
\text { aortic -, n (\%) }\end{array}$ & $\begin{array}{l}\text { Pelvic +/para- } \\
\text { aortic }+, \mathrm{n}(\%)\end{array}$ & $\begin{array}{l}\text { Pelvic-/para- } \\
\text { aortic +, n (\%) }\end{array}$ & $\begin{array}{l}\text { Pelvic +/para- } \\
\text { aortic x, n (\%) }\end{array}$ & $\begin{array}{l}\text { Pelvic } \mathrm{x} / \text { para- } \\
\text { aortic }+, \mathrm{n}(\%)\end{array}$ \\
\hline Serous & $30(23.44)$ & $16(12.5)$ & 41 & $13(10.16)$ & $9(7.05)$ & $1(0.78)$ \\
\hline Mucinous & $2(1.56)$ & $0(0.0)$ & $(32.03)$ & $0(0.0)$ & $1(0.78)$ & $0(0.0)$ \\
\hline Clear cell & $0(0.0)$ & $0(0.0)$ & $0(0.0)$ & $1(0.78)$ & $0(0.0)$ & $0(0.0)$ \\
\hline Endometrioid & $0(0.0)$ & $1(0.78)$ & $2(1.56)$ & $3(2.34)$ & $2(1.56)$ & $0(0.0)$ \\
\hline Undifferentiated & $1(0.78)$ & $0(0.0)$ & $2(1.56)$ & $0(0.0)$ & $1(0.78)$ & $1(0.78)$ \\
\hline
\end{tabular}

Nodal involvement: -, negative; +, positive; x, unknown. FIGO, international federation of gynecology and obstetrics.

Table IV. Prognostic effect (OS and PFS) of the histological subtype (serous vs. non-serous) and lymph node metastatic status (N0 vs. N+) in 129 patients with FIGO stage IIIC/IV primary ovarian cancer.

\begin{tabular}{|c|c|c|c|c|}
\hline Nodal status & PFS, months $(95 \% \mathrm{CI})$ & P-value & OS, months $(95 \% \mathrm{CI})$ & P-value \\
\hline \multicolumn{5}{|l|}{ NO } \\
\hline Serous, $n=29$ & $13.27(11.8-14.72)$ & & $25.97(21.85-30.1)$ & \\
\hline Othera, $n=3$ & $15.83(13.01-15.8)$ & & $16.0(11.8-20.2)$ & \\
\hline $\mathrm{N}+$ & & N.S. & & 0.043 \\
\hline Serous, $n=81$ & $15.433(13.071-17.8)$ & & $47.97(13.9-48.8)$ & \\
\hline Other $^{\mathrm{a}}, \mathrm{n}=16$ & $12.167(10.3-14.04)$ & & $25.7(11.5-39.8)$ & \\
\hline
\end{tabular}

${ }^{a}$ Other: mucinous, clear cell, endometrioid and undifferentiated. OS, overall survival; PFS, progression-free survival; CI, confidence interval; FIGO, international federation of gynecology and obstetrics; N.S., non-significant.

cell subtype, 1 exhibited combined involvement of the pelvic and para-aortic nodes and 1 had involvement of the para-aortic nodes alone (Table III).

A significant positive effect on OS was observed for patients with serous cancers and nodal metastases, compared with node-negative and non-serous cancers $(\mathrm{P}=0.043)$; there was no significant difference in terms of PFS (Table IV).

\section{Discussion}

Complete cytoreduction ( $\mathrm{R}=0 \mathrm{~mm})$, compared with $\mathrm{R}>0 \mathrm{~mm}$ $-\leq 1 \mathrm{~cm}$, exerts a significantly better prognostic effect on PFS and $O S$ in advanced ovarian cancer (6-8). The relevance of lymphadenectomy in the primary surgical management of ovarian cancer remains unclear $(9,10)$ and is currently investigated in the prospective LION study (AGO-Ovar). Results from randomised controlled studies are currently lacking (11,12). The randomised trial of Panici et al revealed a positive effect of systemic lymphadenectomy on PFS compared with resection of bulky nodes in optimally cytoreduced patients, but no effect on OS (2-4). Therefore, pelvic and para-aortic lymphadenectomy following optimal cytoreduction is recommended, with a positive prognostic effect on primary advanced ovarian cancer $(11,13)$. By contrast, another report showed a significant positive prognostic effect of systematic lymphadenectomy on OS in advanced ovarian cancer (4).
Overall, previous studies reported a rate of $\sim 45-60 \%$ nodal involvement in advanced ovarian cancer $(2,3,9,14)$. In the present study, collective nodal metastases were detected in $74.62 \%$ of the cases (Table I). Involvement of the pelvic and/or para-aortic region was also described $(2,3,9)$, which was confirmed in our study (Table I). Thus, accurate surgical staging, including lymphadenectomy, reliably detects the true extent of the disease with detection of occult nodal metastases.

The prognostic relevance of nodal metastases in primary ovarian cancer remains unclear (4). It was previously reported that the impact of lymph node metastasis on prognosis decreases with an increase of residual tumour mass (15). As there are several risk factors for ovarian cancer, it remains questionable whether lymphadenectomy in advanced ovarian cancer improves prognosis. The results of the ongoing prospective LION study (AGO-Ovar) may help to assess the validity of lymphadenectomy as part of the treatment strategy in optimally cytoreduced patients.

Additionally, the association of nodal metastasis with clinicopathological parameters must be investigated.

Di Re et al reported that the incidence of metastatic lymph nodes significantly increased with advanced stage, serous histology and greater amount of residual tumour (10). On multivariate analysis, histological grade 3 , serous and endometrioid histology, were found to be independent predictors of lymph node metastasis in advanced ovarian cancer (Table II). However, even in the less common subtype of non-serous 
cancers, $84.2 \%$ of the patients with advanced ovarian cancer had nodal metastases (Table II).

Since ovarian cancer is known to spread simultaneously intra- and retroperitoneally, the presence of tumour spreading mainly through the lymphatic channels without intraperitoneal dissemination suggests that such tumours may be associated with a favourable biological behaviour (16). A previous study described that ovarian serous carcinoma patients with only extrapelvic peritoneal involvement have a more favorable survival compared with those with extrapelvic peritoneal involvement and lymph node metastases (17). Therefore, the extent to which lymphadenectomy may be considered as reasonable has yet to be determined, and it may depend on the histological findings.

It has been previously described that nodal metastases to both the pelvic and para-aortic nodes, as well as to the pelvic or para-aortic region alone, may be found in advanced ovarian cancer $(3,12,18)$, as was observed in our study (Table I). Both the pelvic and para-aortic nodes were affected in $35.9 \%$ of the patients in our study, with isolated involvement of the pelvic or para-aortic nodes detected in $13.3 \%$ of the patients (Table I).

Serous cancers were associated with all distribution patterns of nodal involvement, although most frequently combined involvement of the pelvic and para-aortic region was observed (Table III). Other histological subtypes, such as endometrioid and mucinous cancers, are associated with metastases to the pelvic and para-aortic lymph nodes. Patients with the endometrioid subtype exhibited all patterns of lymph node metastasis (Table III). The rare mucinous subtype only showed a predilection for the pelvic nodes, without involvement of the para-aortic nodes (Table III). Lymphadenectomy leads to accurate staging with detection of occult node metastases. In our study there was a significant effect on OS in serous cancers with nodal metastases compared with node-negative patients and non-serous cancers ( $\mathrm{P}=0.043$; Table IV). The removal of positive lymph nodes in the pelvic or para-aortic region may explain the positive prognostic effect of lymphadenectomy in advanced-stage disease $(3,10,19)$. This effect is possibly caused by affected nodes in the para-aortic region, which are not removed by pelvic lymphadenectomy alone. This fact supports the hypothesis that adequate staging may be achieved by performing lymphadenectomy (19). Chang et al described involvement of para-aortic nodes and recommended a para-aortic lymphadenectomy up to the level of the renal vessels, which may detect occult metastases and be helpful in tailoring appropriate adjuvant treatment, as well as providing useful prognostic information (20).

Our findings suggest that pelvic and para-aortic lymphadenectomy in advanced ovarian cancer leads to adequate staging and may detect occult node metastases. Consequently, the effect of systematic lymphadenectomy on prognosis in advanced ovarian cancer remains unknown, due to the lack of prospective randomised controlled studies.

Prospective studies are required to investigate the prognostic effect of lymphadenectomy in cases with advanced ovarian cancer depending on histology and distribution pattern of nodal metastases. The stratification of this subpopulation of node-positive epithelial ovarian cancer based on nodal burden may be of significant prognostic value, and it may be considered in future staging and aid with management decisions.
Optimal cytoreduction is the main intention of primary surgery in advanced ovarian cancer, with a significant positive effect on prognosis. There is a need to investigate the risk factors and metastatic patterns of such patients in a multicenter analysis. More extensive lymphadenectomy appears to play an important role through achieving accurate staging by detection of occult metastases. Prospective studies are required to investigate the prognostic effect of lymphadenectomy on advanced ovarian cancer depending on histology and distribution pattern of nodal metastases.

\section{References}

1. Haller H, Mamula O, Krasevic M, Rupcic S, Fischer AB, Eminovic S, Manestar M and Perovic D: Frequency and distribution of lymph node metastases in epithelial ovarian cancer. Int J Gynecol Cancer 21: 245-250, 2011.

2. Takeshima N, Hirai Y, Umayahara K, Fujiwara K, Takizawa K and Hasumi K: Lymph node metastasis in ovarian cancer: Difference between serous and non-serous primary tumors. Gynecol Oncol 99: 427-431, 2005.

3. Panici PB, Maggioni A, Hacker N, Landoni F, Ackermann S, Campagnutta E, Tamussino K, Winter R, Pellegrino A, Greggi S, et al: Systematic aortic and pelvic lymphadenectomy versus resection of bulky nodes only in optimally debulked advanced ovarian cancer: A randomized clinical trial. J Natl Cancer Inst 97: 560-566, 2005.

4. Pereira A, Pérez-Medina T, Magrina JF, Magtibay PM, Millan I and Iglesias E: The role of lymphadenectomy in node-positive epithelial ovarian cancer. Int J Gynecol Cancer 22: 987-992, 2012.

5. Prat J; FIGO Committee on Gynecologic Oncology: Staging classification for cancer of the ovary, fallopian tube, and peritoneum. Int J Gynaecol Obstet 124: 1-5, 2014.

6. Abe A, Furumoto H, Irahara M, Ino H, Kamada M, Naka O Sasaki M, Kagawa T, Okitsu O and Kushiki N: The impact of systematic para-aortic and pelvic lymphadenectomy on survival in patients with optimally debulked ovarian cancer. J Obstet Gynaecol Res 36: 1023-1030, 2010.

7. du Bois A, Reuss A, Pujade-Lauraine E, Harter P, Ray-Coquard I and Pfisterer J: Role of surgical outcome as prognostic factor in advanced epithelial ovarian cancer: A combined exploratory analysis of 3 prospectively randomized phase 3 multicenter trials: By the arbeitsgemeinschaft gynaekologische onkologie studiengruppe ovarialkarzinom (AGO-OVAR) and the groupe d'investigateurs nationaux pour les etudes des cancers de l'Ovaire (GINECO). Cancer 115: 1234-1244, 2009.

8. Wimberger P, Lehmann N, Kimmig R, Burges A, Meier W and Du Bois A; Arbeitsgemeinschaft Gynaekologische Onkologie Ovarian Cancer Study Group: Prognostic factors for complete debulking in advanced ovarian cancer and its impact on survival. An exploratory analysis of a prospectively randomized phase III study of the Arbeitsgemeinschaft gynaekologische onkologie ovarian cancer study group (AGOOVAR). Gynecol Oncol 106: 69-74, 2007.

9. Aletti GD, Dowdy S, Podratz KC and Cliby WA: Role of lymphadenectomy in the management of grossly apparent advanced stage epithelial ovarian cancer. Am J Obstet Gynecol 195: 1862-1868, 2006.

10. di Re F, Baiocchi G, Fontanelli R, Grosso G, Cobellis L, Raspagliesi F and di Re E: Systematic pelvic and paraaortic lymphadenectomy for advanced ovarian cancer: Prognostic significance of node metastases. Gynecol Oncol 62: 360-365, 1996.

11. Carnino F, Fuda G, Ciccone G, Iskra L, Guercio E, Dadone D and Conte PF: Significance of lymph node sampling in epithelial carcinoma of the ovary. Gynecol Oncol 65: 467-472, 1997.

12. Kim HS, Ju W, Jee BC, Kim YB, Park NH, Song YS, Kim SC, Kang SB and Kim JW: Systematic lymphadenectomy for survival in epithelial ovarian cancer: A meta-analysis. Int J Gynecol Cancer 20: 520-528, 2010.

13. Onda T, Yoshikawa H, Yasugi T, Mishima M, Nakagawa S, Yamada M, Matsumoto K and Taketani Y: Patients with ovarian carcinoma upstaged to stage III after systematic lymphadenctomy have similar survival to Stage I/II patients and superior survival to other Stage III patients. Cancer 83: 1555-1560, 1998. 
14. Harter P, Gnauert K, Hils R, Lehmann TG, Fisseler-Eckhoff A Traut A and du Bois A: Pattern and clinical predictors of lymph node metastases in epithelial ovarian cancer. Int J Gynecol Cancer 17: 1238-1244, 2007.

15. Balbi G, Manganaro MA, Monteverde A, Landino I, Franzese C and Gioia F: Ovarian cancer: Lymphnodemetastases. Eur J Gynaecol Oncol 30: 289-291, 2009.

16. Suh DH, Kim TH, Kim JW, Kim SY, Kim HS, Lee TS, Chung HH, Kim YB, Park NH and Song YS: Improvements to the FIGO staging for ovarian cancer: Reconsideration of lymphatic spread and intraoperative tumor rupture. J Gynecol Oncol 24: 352-358, 2013.

17. Bakkar R, Gershenson D, Fox P, Vu K, Zenali M and Silva E: Stage IIIC ovarian/peritoneal serous carcinoma: A heterogeneous group of patients with different prognoses. Int J Gynecol Pathol 33: 302-308, 2014
18. Kim HS, Ju W, Jee BC, Kim YB, Park NH, Song YS, Kim SC, Kang SB and Kim JW: Systematic lymphadenectomy for survival in epithelial ovarian cancer: A meta-analysis. Int J Gynecol Cancer 20: 520-528, 2010.

19. Scarabelli C, Gallo A, Visentin MC, Canzonieri V, Carbone A and Zarrelli A: Systematic pelvic and para-aortic lymphadenectomy in advanced ovarian cancer patients with no residual intraperitoneal disease. Int J Gynecol Cancer 7: 18-26, 1997.

20. Chang SJ, Bristow RE and Ryu HS: Analysis of para-aortic lymphadenectomy up to the level of the renal vessels in apparent early-stage ovarian cancer. J Gynecol Oncol 24: 29-36, 2013. 